\title{
Communications
}

\section{Analog Circuit for Real-Time Computation of Respiratory Mechanical Impedance in Sleep Studies}

Ramon Farré,* Mar Rotger, Josep M. Montserrat, and Daniel Navajas

\begin{abstract}
The aim of this work was to develop a low-cost circuit for real-time analog computation of the respiratory mechanical impedance in sleep studies. The practical performance of the circuit was tested in six patients with obstructive sleep apnea. The impedance signal provided by the analog circuit was compared with the impedance calculated simultaneously with a conventional computerized system. We concluded that the low-cost analog circuit developed could be a useful tool for facilitating the real-time assessment of airway obstruction in routine sleep studies.
\end{abstract}

Index Terms-Airway resistance, forced oscillation, monitoring, sleep apnea.

\section{INTRODUCTION}

The forced oscillation technique (FOT) allows us to explore the mechanical properties of the respiratory system with a procedure which is not invasive and does not require patient cooperation [1]. This technique is based on superimposing a high-frequency smallamplitude pressure oscillation at the entrance of the airway and on computing the mechanical impedance of the respiratory system $(Z)$ from the flow-pressure relationship. The FOT has been widely used to measure $Z$ in a variety of clinical fields such as bronchoconstriction [2] and brochodilation [3] tests, anesthesia [4], and intensive care [5]. As has been shown recently [6], [7], [8], FOT is also useful in assessing inspiratory and expiratory airway obstruction in the recurrent upper-airway collapses found in patients with obstructive sleep apnea/hypopnea (OSA).

Sleep studies require the acquisition and storage of a variety of signals characterizing the sleep status of the patient (thermistors, strain gauges, inductance plethysmography, esophageal pressure, oxygen saturation, etc.) [9], [10]. The signal recording is commonly carried out in special purpose massive-data acquisition and storage systems given that a considerable amount of data is involved in sleep studies. The conventional polysomnographic systems usually do not allow signal processing other than amplification and filtering and, therefore, an analog $Z$ signal is required for real-time assessment of airway obstruction by FOT in sleep studies. At present, an analog $Z$ signal may be obtained by means of a special purpose computerized FOT system allowing real-time computation of $Z$ and incorporating a D/A board to generate the analog $Z$ signal. In order to obviate the need for such a computerized FOT system, which is complex and expensive, our aim was to design and evaluate a low-cost analog circuit for real-time computation of respiratory impedance in monitoring airway obstruction in routine sleep studies.

Manuscript received July 26, 1996; revised April 18, 1997. This work was supported in part by Comisión Interministerial de Ciencia y Tecnología under Grant SAF96-0076. Asterisk indicates corresponding author.

*R. Farré is with the Laboratori Biofísica i Bioenginyeria, Facultat de Medicina, Casanova 143, Barcelona E-08036 Spain (e-mail: farre@medicina.ub.es).

M. Rotger and D. Navajas are with the Lab. Biofísica i Bioenginyeria, Facultat de Medicina, Barcelona E-08036 Spain.

J. M. Montserrat is with the Servei de Pneumologia (Hospital Clinic), Facultat de Medicina, Universitat de Barcelona, Barcelona E-08036 Spain.

Publisher Item Identifier S 0018-9294(97)07411-9.

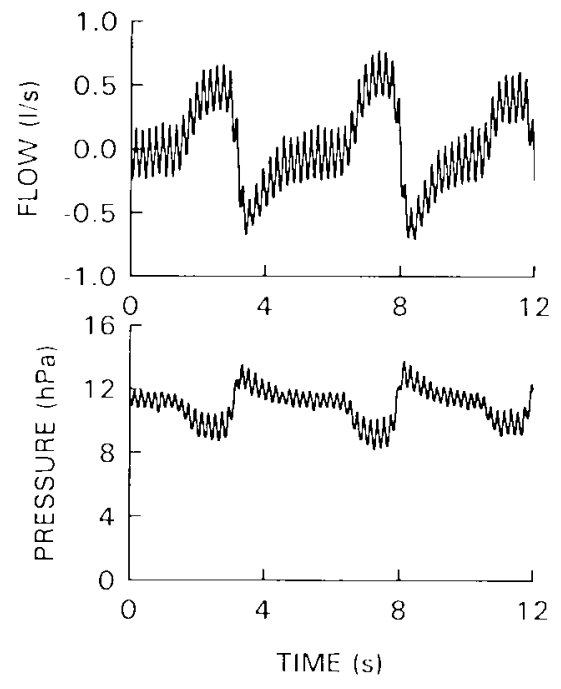

Fig. 1. Example of the pressure and flow signals recorded at the nasal mask when applying FOT to a patient with OSA subjected to CPAP.

\section{DesCRIPTION OF THE CIRCUIT}

An example of the pressure $(P n)$ and flow $\left(V^{\prime} n\right)$ signals recorded during application of FOT in a patient with OSA treated with a continuous positive airway pressure (CPAP) of $12 \mathrm{hPa}\left(1 \mathrm{hPa}=100 \mathrm{~N} / \mathrm{m}^{2}\right)$, which is the conventional therapy for these patients, is shown in Fig. 1. The 5-Hz oscillation components of $P n$ and $V^{\prime} n$ are superimposed to the CPAP and to the low-frequency (period of $\approx 5 \mathrm{~s}$ ) breathing flow respectively. Overall airway obstruction is assessed in FOT by the amplitude of respiratory mechanical impedance $(|Z|)$, which is the quotient between the amplitudes of the forced oscillation components of pressure and flow. The analog circuit implemented for realtime computation of $|Z|$ is shown in Fig. 2. First, signals $P n$ and $V^{\prime} n$ are independently preprocessed to obtain the amplitude of their forced oscillation components. To this end, each signal is highpass filtered ( $5 \mathrm{~Hz}, 3 \mathrm{~dB}$ ) to eliminate the low-frequency components due to patient's breathing and subsequently the signal amplitude is adequately increased. Then, the signal is fed into an active full-wave rectifier. The rectified signal is lowpass filtered $(5 \mathrm{~Hz}, 3 \mathrm{~dB})$ to eliminate the harmonic components with frequencies multiple of $10 \mathrm{~Hz}$ induced by the rectifier and to obtain the dc component. Second, the amplitudes of the forced oscillation components of pressure and flow are divided to obtain $|Z|$. Before division, the amplitudes of the preprocessed pressure and flow signals are modified to fulfill the input requirements of a general purpose low-cost analog divider (MPY100, Burr-Brown). Finally, the output of the divider is lowpass filtered $(1.5 \mathrm{~Hz}, 3 \mathrm{~dB})$ to obtain a smooth analog impedance signal $\left(|Z|_{a}\right)$ while maintaining the phasic changes during the breathing cycle. All the highpass and lowpass filters in the system are Butterworth-type, eight-poles, Sallen-Key configuration. The gain stages (Fig. 2) allowed offset compensation. Owing to the filtering involved in the computation of $|Z|_{a}$, the delay between this signal and the recorded $P n$ and $V^{\prime} n$ is $0.89 \mathrm{~s}$.

\section{Assessment IN SLeEP StUdies}

The circuit designed for analog real-time computation of respiratory mechanical impedance was practically tested by using real 


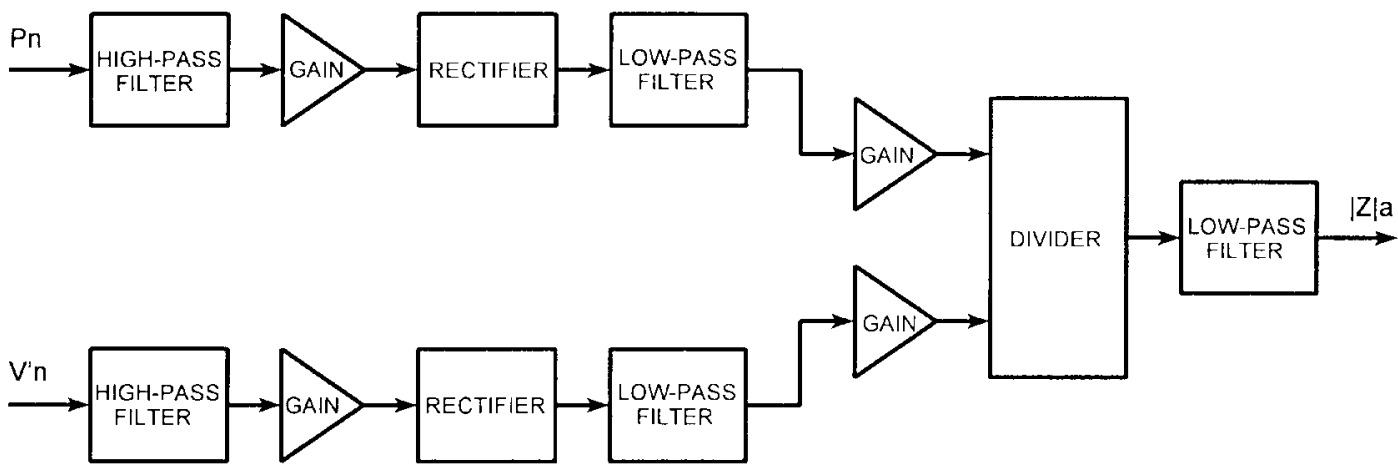

Fig. 2. Diagram of the analog circuit for real-time computation of respiratory mechanical impedance. $P n$ : nasal pressure, $V^{\prime} n$ : nasal flow, $|Z|_{a}$ : amplitude of mechanical impedance. All the filters were Butterworth-type, eight-poles. The cutoff frequency was $5 \mathrm{~Hz}(3 \mathrm{~dB})$, except in the final filter where it was $1.5 \mathrm{~Hz}(3 \mathrm{~dB})$.

signals recorded in patients [6]. The study was carried out during CPAP titration with full polysomnography on six male patients with OSA. Forced oscillation $(5 \mathrm{~Hz})$ was applied during sleep for $\mathrm{CPAP}=4 \mathrm{hPa}$ and for the optimal CPAP in each patient $(10-14$ $\mathrm{hPa}$ ) determined by absence of arousals, normal sleep architecture, and awake tidal breathing esophageal pressure. $P n$ and $V^{\prime} n$ were recorded at the nasal mask by a piezoresistive pressure transducer (176 PC, Honeywell) and a Fleisch-type pneumotachograph with a differential pressure transducer [ $\pm 2 \mathrm{hPa}, \mathrm{LCVR}$, Celesco] [7]. These $P n$ and $V^{\prime} n$ signals were fed to the analog circuit designed to compute $|Z|_{a}$ (Fig. 2) and were simultaneously sampled at $100 \mathrm{~Hz}$ by a computerized system to calculate impedance by digital signal processing $\left(|Z|_{d}\right)$ according to the procedures usually employed in the field of FOT and described in detail in [7]. Briefly, a movingaverage filter with a window length of one FOT period $(0.2 \mathrm{~s})$ was used to obtain the oscillatory components of pressure and flow from the sampled $P n$ and $V^{\prime} n$ [7]. $|Z|_{d}$ was calculated point-by-point from the fast Fourier transform (FFT) of the filtered pressure and flow signals computed on a cycle-per-cycle basis (0.2 s) [7], [11] and smoothed by a moving-average filter with a window length of $0.4 \mathrm{~s}$. As we used phaseless moving-average digital filters, $|Z|_{d}$ was undelayed as regards the recorded $P n$ and $V^{\prime} n$ signals. Respiratory impedance computed with the designed analog circuit $\left(|Z|_{a}\right)$ and impedance computed digitally $\left(|Z|_{d}\right)$, according to conventional procedures were compared. To this end, for each patient and CPAP we computed the mean impedances $\left(|Z|_{d}\right.$ and $\left.|Z|_{a}\right)$ at midinspiration and midexpiration from three consecutive breathing cycles.

\section{RESUlTS}

Fig. 3 shows representative examples of the results obtained when comparing $|Z|_{a}$ and $|Z|_{d}$ in one of the patients for CPAP of $4 \mathrm{hPa}$ [Fig. 3(a)] and $12 \mathrm{hPa}$ [Fig. 3(b)]. In this figure the small time delay between both signals due to the filtering involved in the analog and digital procedures to compute impedance was corrected. At $\mathrm{CPAP}=4 \mathrm{hPa}$ the patient developed sleep apneas, as clearly indicated by the breathing flow signal shown in the lower panels: an obstructive apnea lasted for the first $\approx 10 \mathrm{~s}$, then an arousal occurred and the patient breathed normally during two cycles (at time $\approx 10-20 \mathrm{~s}$ ), and finally hypopnea (at time $\approx 20-30 \mathrm{~s}$ ) and apnea developed before the following arousal (at time $\approx 37 \mathrm{~s}$ ). Consistently, respiratory impedance amplitude was high $(38 \mathrm{hPa} \cdot \mathrm{s} / \mathrm{l})$ during the apneas, markedly decreased down to $8 \mathrm{hPa} \cdot \mathrm{s} / \mathrm{l}$ during the normal breathing and rapidly changed from the maximum to the minimum values during the hypopnea. The right panel in this figure shows the impedance amplitude and the breathing flow in the same patient when submitted to his optimal CPAP $=12 \mathrm{hPa}$. The breathing flow exhibited a normal pattern and impedance remained at low values
$(<8 \mathrm{hPa} \cdot \mathrm{s} / \mathrm{l})$ with periodic fluctuations according to the phase of the breathing cycle (Fig. 3). In this representative example the realtime impedance computed by means of the designed analog circuit (solid lines) was almost indistinguishable from impedance computed digitally (dashed lines). The only differences between analog and digital impedances appeared during the transient unstable breathing corresponding to the patient's arousal following an apnea [at time $\approx 15 \mathrm{~s}$ in Fig. 3(a)]. The results obtained when comparing $|Z|_{a}$ and $|Z|_{d}$ in all the patients and CPAP's both during inspiration and expiration are shown in Fig. 4. Confirming the results in the example in Fig. 3, we found an extremely good correspondence between the analogically and the digitally computed impedances. The linear regression equation of these data was $|Z|_{a}=1.02 \cdot|Z|_{d}-0.14$ and the coefficient of correlation was $r^{2}=0.998$.

\section{DisCUSSION}

To design the analog circuit for real-time computation of respiratory mechanical impedance we implemented a signal processing similar to the one carried out in the digital procedures used in the field of FOT [7], [11], [12]. The first stage in the system (Fig. 2) was devoted to separate the forced oscillation from the breathing components. Given that the forced oscillation frequency $(5 \mathrm{~Hz})$ was very close to the frequency band associated with the breathing signals, especially in the transient events related to airway occlusions in sleep apnea, we implemented a filter (eight-poles, Butterworth) optimizing flatness of passband and rolloff rate. With such a filter, we were able to obtain the forced oscillation component with an efficiency similar to the one achieved with a digital moving average filter with a window length matching the oscillatory period. To analogically mimic the conventional digital FFT computation to obtain the amplitude of oscillation with a simple and low-cost stage we implemented an active full-wave rectifier. This stage avoided the diode drops of passive rectifiers. For a sinusoidal input of $5 \mathrm{~Hz}$, the rectifier provided an output signal with a dc component proportional to the amplitude of the sinusoidal input and with harmonic components with frequencies multiple of $10 \mathrm{~Hz}$. These high-frequency components were eliminated with a lowpass filter with a cutoff frequency $(5 \mathrm{~Hz})$ which did not affect the time variation of the dc component associated with the changes of impedance within the breathing cycle. The low-cost analog divider we employed proved to be sufficiently reliable to compute quotients within the ranges potentially found in practical applications (Fig. 4). Finally, the analog impedance signal was smoothed by a lowpass filter which played the role of the final digital moving average filter used in the computerized FOT system.

The low-cost circuit designed was tested under very demanding conditions from the viewpoint of the filtering requirements in order to improve the signal-to-noise ratio [13], [14]. Indeed, we used a 

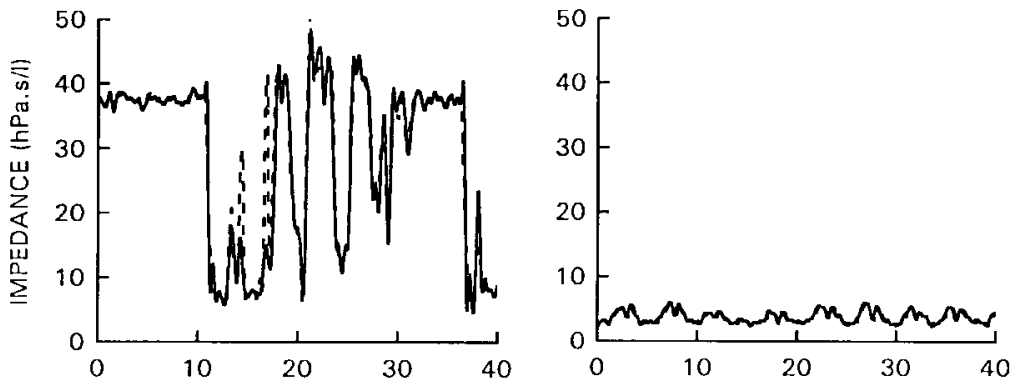

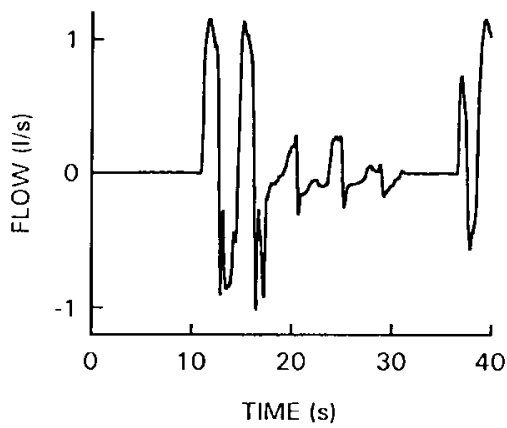

(a)

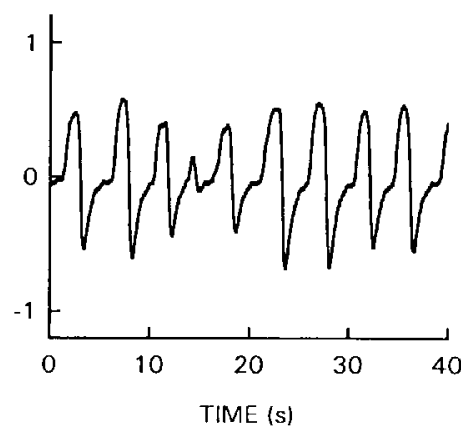

(b)

Fig. 3. Representative example of respiratory impedance in a patient with OSA at (a) CPAP $=4 \mathrm{hPa}$ and (b) at CPAP $=12 \mathrm{hPa}$. Top: Impedances computed by the analog circuit (solid lines) and with the computerized system (dashed lines). Solid and dashed lines are almost indistinguishable, which indicates that analog and digital computations of impedance provide practically the same result. Bottom: The breathing flow was digitally filtered to eliminate the forced oscillation component.

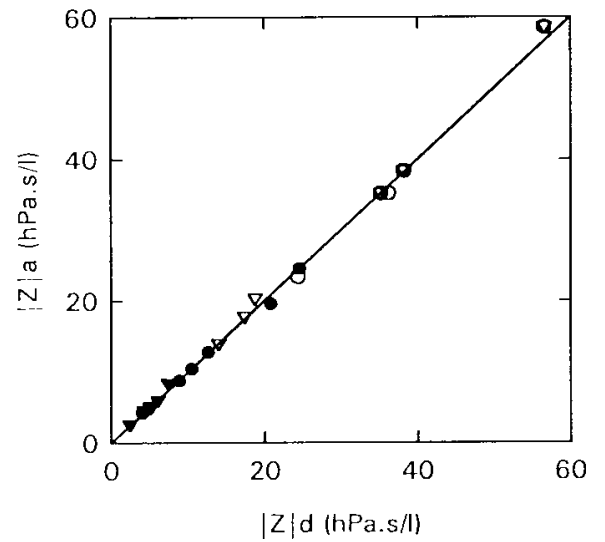

Fig. 4. Respiratory impedance computed by the analog circuit $\left(|Z|_{a}\right)$ and by a computerized system $\left(|Z|_{d}\right)$ for the six patients with OSA during inspiration $(\circ)$ and expiration $(\nabla)$ for $\mathrm{CPAP}=4 \mathrm{hPa}$ and during inspiration $(\bullet)$ and expiration $(\boldsymbol{\nabla})$ for the optimal CPAP.

forced oscillation frequency $(5 \mathrm{~Hz})$ which is in the lower limit of the frequency range commonly employed in applications of FOT in spontaneously breathing or mechanically ventilated patients (5-30 $\mathrm{Hz}$ ) [1]. The main difficulty of operating at such a low frequency is that it overlaps the frequency band of the breathing signals and, therefore, efficient filters such as the ones used in this work are required to separate signal and noise. Nevertheless, in case the analog circuit was intended to operate with a forced oscillation frequency higher than $5 \mathrm{~Hz}$ the design could be modified by reducing the rolloff rate of the filters (i.e., the number of its poles), thereby simplifying its implementation. Moreover, the circuit could be further simplified if the interest was not focused on the fast impedance changes occurring within the breathing cycle. In such a case, the cutoff frequency of the filters that follow the rectifier (Fig. 2) could be reduced and the final lowpass filter applied to the quotient signal could be suppressed. It is worth noting that in addition to the specific application for which the circuit was implemented and tested, the designed device could be potentially useful to spread the clinical application of FOT, especially in situations, such as intensive care [12], requiring realtime monitoring of respiratory impedance to assess the changes of $|Z|$ within the breathing cycle. Indeed, its use may considerably simplify the complexity and cost of application of FOT since the analog circuit may substitute the computerized elements which perform digital signal processing in a FOT system.

In conclusion, the practical performance of the designed circuit was assessed when applied in patients with OSA during CPAP titration. The test covered the full range of possible impedance values (minimum at normalized CPAP and maximum during obstructive apneas, respectively) and time-pattern of signals (smooth normal breathing, hypopneas, and transient changes associated with sudden airway opening and closure in apneas and arousals). The results obtained (Figs. 3 and 4) showed that the analog circuit is a reliable device which given to its low cost and simplicity could be a useful tool for facilitating the application of FOT to monitor airway obstruction with conventional polysomnographic systems in routine sleep studies.

\section{ACKNOWLEDGMENT}

The authors wish to thank M. A. Rodriguez for his technical assistance.

\section{REFERENCES}

[1] R. Peslin and J. J. Fredberg, "Oscillation mechanics of the respiratory system," in Handbook of Physiology, Vol. 3.. Bethesda, MD: Amer. Physiol. Soc., sect. 3, pt. I, 1986.

[2] J. A. Van Noord, J. Clement, K. P. Van de Woestijne, and M. Demedts, "Total respiratory resistance and reactance as a measurement of response to bronchial challenge with histamine," Amer. Rev. Respir. Dis., vol. 139, pp. 921-926, 1989.

[3] J. A. Van Noord, J. Smeets, J. Clement, K. P. Van de Woestijne, and M. Demedts, "Assessment of reversibility of airway obstruction," Amer. J. Respir. Crit. Care. Med., vol. 150, pp. 551-554, 1994.

[4] D. Navajas, R. Farré, J. Canet, M. Rotger, and J. Sanchis, "Respiratory input impedance in anesthetized paralyzed patients," J. Appl. Physiol., vol. 69, pp. 1372-1379, 1990.

[5] R. Farré, M. Ferrer, M. Rotger, and D. Navajas, "Servocontrolled generator to measure respiratory impedance from 0.25 to $26 \mathrm{~Hz}$ in 
ventilated patients at different PEEP levels," Eur. Respir. J., vol. 8, pp. 1222-1227, 1995.

[6] D. Navajas, R. Farré, M. Rotger, and J. M. Montserrat, "Assessment of airway obstruction by means of the forced oscillation technique during application of CPAP in patients with SAS," Amer. J. Respir. Crit. Care. Med., vol. 153, no. 4, p. A772, 1996.

[7] R. Farré, R. Peslin, M. Rotger, and D. Navajas, "Inspiratory dynamic obstruction detected by forced oscillation during CPAP. A model study," Amer. J. Respir. Crit. Care. Med., vol. 155, pp. 952-956, 1997.

[8] R. Farré, M. Rotger, J. M. Montserrat, and D. Navajas, "System to generate forced oscillation superimposed to continuous positive airway pressure," Eur. Respir. J., vol. 10, pp. 1349-1353, 1997.

[9] S. A. Keenan, "Polysomnographic techniques: An overview," in Sleep Disorders Medicine, S. Chokroverty, Ed. Stoneham, MA,: Butterworth-Heinemann, ch. 6, pp. 79-94, 1994.

[10] M. A. Carskadon and A. Rechtschaffen, "Monitoring and staging human sleep," in Principles and Practice of Sleep Medicine, M. H. Kryger, T. Roth, and W. C. Dement, Eds. Philadelphia, PA: Saunders, ch. 89, pp. 943-960, 1994.

[11] J. G. Horowitz, S. D. Siegel, F. P. Primiano, Jr., and E. H. Chester, "Computation of respiratory impedance from forced sinusoidal oscillations during breathing," Computers, Biomed. Res., vol. 16, pp. 499-521, 1983.

[12] R. Peslin, J. F. da Silva, C. Duvivier, and F. Chabot, "Respiratory mechanics studied by forced oscillations during artificial ventilation," Eur. Respir. J., vol. 6, pp. 772-784, 1993.

[13] D. Navajas, R. Farré, M. Rotger, and R. Peslin, "A new estimator to minimize the error due to breathing in the measurerent of respiratory impedance," IEEE Trans. Biomed. Eng., vol. 35, pp. 1001-1005, 1988.

[14] R. Farré, M. Rotger, and D. Navajas, "Time-domain digital filter to improve signal-to-noise ratio in respiratory impedance measurements," Med. Biol. Eng. Comput., vol. 29, pp. 18-24, 1991.

\section{Microelectrode Arrays for Electrophysiological Monitoring of Hippocampal Organotypic Slice Cultures}

P. Thiébaud,* N. F. de Rooij, M. Koudelka-Hep, and L. Stoppini

\begin{abstract}
A three-dimensional platinum (Pt) microelectrode array embedded on a micromachined silicon (Si) substrate (porosity of $13 \%$, via hole diameter of $40 \mu \mathrm{m}$ ) has been developed. Electrodes are 35- $\mu \mathrm{m}$ wide and $20-\mu \mathrm{m}$ high, spaced $200 \mu \mathrm{m}$ apart and arranged in an elliptic geometry. Integrated within a microperfusion chamber, the devices were used for stimulation and recording experiments of hippocampal slice cultures over a period of several days.
\end{abstract}

Index Terms - Electrophysiology, microelectrode array, organotypic cultures, perforated substrate.

\section{INTRODUCTION}

The development of microelectrode arrays embedded on a solid substrate for extracellular monitoring of electrophysiological activities of cell cultures is a very active research area motivated

Manuscript received January 10, 1997; revised June 16, 1997. The work of L. Stoppini was supported in part by the FNRS under Grant 31-49799.96. Asterisk indicates corresponding author.

*P. Thiébaud is with the Institute of Microtechnology, University of Neuchâtel, rue Jaquet-Droz 1, 2007 Neuchâtel, Switzerland (e-mail: pierre thiebaud@imt.unine.ch).

N. F. de Rooij and M. Koudelka-Hep are with the Institute of Microtechnology, University of Neuchâtel, Jaquet-Droz 1, 2007 Neuchâtel, Switzerland.

L. Stoppini is with the Department of Pharmacology, University of Geneva, 1211 Geneva 4, Switzerland.

Publisher Item Identifier S 0018-9294(97)07404-1. by the increasing importance of electrogenic cell cultures in both fundamental and application oriented research. Numerous designs of planar microelectrode arrays have been developed over the past 25 years [1]-[7] and their suitability for short- to medium-term electrophysiological monitoring has been clearly demonstrated [8].

Hippocampal slice cultures represent an in vitro model which retains the organotypic structure and much of appropriate synaptic circuitry, physiology, and neurotransmitter receptor distribution of the intact hippocampi [9]. When using a perforated substrate, the slice cultures can be maintained under well-controlled in vitro conditions for several weeks, so allowing the neuronal development to closely parallel that in vivo. In this context, photolithographically defined microelectrode arrays on either perforated polymide [10] or micromachined $\mathrm{Si} / \mathrm{Si}_{3} \mathrm{~N}_{4}$ membranes [11] have been investigated. Their respective shortcomings are the long-term instability of the polymide in physiological solutions and the mechanical fragility of the micromachined membranes.

This paper describes the fabrication of a Pt microelectrode array consisting of 20 electrodes, each with an active area of $10 \mu \mathrm{m} \times$ $10 \mu \mathrm{m}$, separated by $200 \mu \mathrm{m}$, embedded on a micromachined silicon substrate. The first aspect of the present work was an improvement of the mechanical stability of the perforated silicon substrate. This has been achieved by using a deep reactive ion etching (DRIE) of a $380-\mu \mathrm{m}$-thick silicon substrate. The second aspect considered in this work was a possible improvement of the cell/microelectrode interface. This has been attempted by the modification of the microelectrodes with a thick bright Pt deposit resulting in a three-dimensional (3-D) electrode structures. These devices were then tested in stimulating and recording experiments of organotypic culture of hippocampal slices over a period of several days.

\section{Materials AND Methods}

\section{A. Microelectrode Array Fabrication}

The devices were fabricated on 3 in, 380- $\mu$ m-thick silicon wafers. First the wafer was passivated with a 100 -nm-thick thermally grown $\mathrm{SiO}_{2}$ and a 150 -nm-thick layer of $\mathrm{Si}_{3} \mathrm{~N}_{4}$ deposited by low-pressure chemical vapor deposition (LPCVD). A 25-nm-thick layer of Ta, used as an adhesion layer was e-gun evaporated in a one pump-down process prior to the evaporation of $125 \mathrm{~nm}$ of $\mathrm{Pt}$. The electrodes were patterned photolithographically using a lift-off process. The top passivation layer consisted of a 250-nm-thick LPCVD $\mathrm{Si}_{3} \mathrm{~N}_{4}$ layer. The $\mathrm{Si}_{3} \mathrm{~N}_{4}$ layer was etched in a $\mathrm{SF}_{6} / \mathrm{O}_{2}$ plasma to open the active electrode areas $(10 \mu \mathrm{m} \times 10 \mu \mathrm{m})$ and the bonding pads. The etching of via holes (40- $\mu \mathrm{m}$ diameter) was performed in two steps. In the first one, the passivation layers were etched using a standard photolithography in a $\mathrm{SF}_{6} / \mathrm{O}_{2}$ plasma for the $\mathrm{Si}_{3} \mathrm{~N}_{4}$ and in a buffered hydrofluoric acid (BHF) solution for the $\mathrm{SiO}_{2}$. For the second step, a photolithography using a 7.5- $\mu$ m-thick AZ 4562 photoresist (Shipley) defining the via holes through the silicon substrate over an area of $3 \mathrm{~mm} \times 4 \mathrm{~mm}$ was carried out prior to a DRIE of the silicon. This process was carried out at Surface Technology Systems, U.K. (STS) using an inductively coupled plasma (ICP) system. Welldefined vertical via holes were obtained all over the wafer surface. Following the wafer dicing, the devices having an overall dimension of $10 \mathrm{~mm} \times 10 \mathrm{~mm}$ were bonded and encapsulated on a custom-made Kapton support.

The platinum electroplating step was performed by cyclic voltammetry [potential range from 0 to $-0.75 \mathrm{~V}$ versus saturated calomel 